\title{
IRREDUCIBLE POLYNOMIAL IDENTITIES IN ANTICOMMUTATIVE ALGEBRAS
}

\author{
SEYMOUR KASS AND WILLIAM G. WITTHOFT ${ }^{1,2}$
}

\begin{abstract}
The methods introduced by J. M. Osborn for isolating those polynomial identities worth studying in commutative algebras are here modified to yield three theorems for anticommutative algebras. The first establishes a practical criterion for the irreducibility of polynomial identities; the others list all canonical polynomials of low degree that are irreducible relative to anticommutativity.
\end{abstract}

Introduction. In the following we shall obtain a list of homogeneous polynomial identities of degree no higher than four that are irreducible in an anticommutative algebra over a field of characteristic different from two. The idea of irreducibility is due to Osborn [2] who was concerned with commutative algebras with unity elements. We shall modify his definitions in the next section and produce a criterion for irreducibility in Theorem 1 which does not call for algebras with unity elements and so allows us to apply his methods to anticommutative algebras. Our results are important because they reduce the study of anticommutative algebras satisfying an identity of degree $\leqq 4$ to the study of those which satisfy one of the identities in Theorem 3. The proof of Theorem 1 below is essentially due to Osborn.

I. Definitions and criterion for irreducibility. Let $H$ be the family of all polynomials $p$ over a field $F$ that are homogeneous in each of several noncommuting and nonassociating indeterminates $x_{i}$. Let the variables in each nonconstant $p$ in $H$ be indexed in the decreasing order of their degree, i.e., if $p$ is homogeneous in each of $k>0$ indeterminates, choose indices so that $p$ is of degree $d_{i}$ in $x_{i}$ for $i=1,2,3$, $\cdots, k$, and $d_{1} \geqq d_{2} \geqq \cdots \geqq d_{k}>0 . H$ is then partitioned into cells, called types, unambiguously designated by $k$-tuples $\left[d_{1}, d_{2}, \cdots, d_{k}\right]$

Received by the editors August 23, 1969.

AMS 1968 subject classifications. Primary 1710, 1760; Secondary 1230.

Key words and phrases. Nonassociative algebras, anticommutative algebras, irreducible polynomial identities, free nonassociative algebra, linearization.

1 The authors were supported in part by NSF Grants GP-9635 and GP-7130, respectively.

2 The authors are grateful to the referee for pointing out some obscurities and inaccuracies in the original draft. 
for various $k>0$ (with the amendment that [0] be taken to designate the type of all constant polynomials). The polynomial $\left(x^{2} y\right) x-x^{2}(y x)$ is, for example, of type $[3,1]$. The family of types is linearly ordered by taking $\left[c_{1}, c_{2}, \cdots, c_{k}\right]<\left[d_{1}, d_{2}, \cdots, d_{m}\right]$ if and only if either $\sum_{1}^{k} c_{i}<\sum_{1}^{m} d_{i}$ or $\sum_{1}^{k} c_{i}=\sum_{1}^{m} d_{i}$ but $d_{j}<c_{j}$ for the lowest index $j$ at which $c_{j} \neq d_{j}$. Thus $[3,1]<[2,2]$. $H$ then becomes partially ordered when we take $p<q$ if and only if type $p<$ type $q$.

It is important to observe how the type of a polynomial is affected by multiplication and composition. It is clear that the type of a product of two nonconstant members of $H$ exceeds that of each factor. As for composition, if $p\left(x_{1}, \cdots, x_{n}\right)$ is in $H$ and $f_{i}$ is any polynomial (not necessarily homogeneous) in indeterminates $x_{1}, x_{2}, \cdots$, then $p\left(x_{1}, \cdots, f_{i}, \cdots, x_{n}\right)$ is a sum of polynomials in $H$ each obtained from $p$ by replacing $x_{i}$ by a term of $f_{i}$. Terms of degree greater than one yield polynomials of higher type than that of $p$, while terms of degree one can lead to polynomials of type higher or lower than or the same as that of $p$. For example, if $p(x, y)=x^{2} y$, of type $[2,1]$, then $p(x+y+z, y)$ is a sum of polynomials of types [3], [2, 1], and $[1,1,1]$. In particular, linearizing a polynomial identity produces an identity involving a polynomial of higher type, while identifying variables in a polynomial lowers its type.

An algebra $A$ over $F$ satisfies $p$ if and only if $p(a)=0 \in A$ for every $a \in A^{k}$, where $k$ is the number of indeterminates in $p$. Similarly, $A$ satisfies a subfamily $S$ of $H$ if and only if $A$ satisfies every $p$ in $S$. For $p$ and $q$ in $H$ we shall say that $p$ implies $q(p \Rightarrow q)$ in case every algebra that satisfies $p$ satisfies $q$. Again, for subfamilies $S$ and $T$ of $H, S \Rightarrow T$ if and only if every algebra that satisfies $S$ satisfies $T$. The zero polynomial and the empty family imply each other but do not imply any other polynomial or family. In case the union of a family $S$ and a single polynomial $p$ implies no polynomial of type lower than $p$ that is not already implied by $S$ alone, we say that $p$ is irreducible with respect to $S$. Thus $p$ is irreducible with respect to $S$ if and only if for every $q$ of lower type than $p$, if there exists an algebra which satisfies $S$ but not $q$ then there exists an algebra which satisfies $\{p\} \cup S$ but not $q$.

Among the polynomials implied by a polynomial $p$ are those obtained from $p$ by partial or full linearization or by identification of variables. If $p$ is irreducible with respect to $S$, then all polynomials so obtained from $p$ are implied by $S$. The fundamental theorem about irreducibility tells us that there are no other processes to worry about.

Theorem 1. A polynomial $p$ in $H$ is irreducible with respect to a subfamily $S$ of $H$ if and only if, for each $q \in H$, if $q<p$ and $q$ is obtained 
from $p$ by identifying variables in $p$ or in some partial linearization of $p$, then $S \Rightarrow q$.

Proof. Suppose all the polynomials so obtained from $p$ are implied by $S$. Let $q$ be a homogeneous polynomial of lower type than $p$, and let $A$ be an algebra that satisfies $S$ but not $q$. We shall prove $p$ irreducible with respect to $S$ by constructing an algebra $B$ that satisfies $\{p\} \cup S$ but not $q$.

Say $q$ is of type $\left[t_{1}, t_{2}, \cdots, t_{r}\right]$ and degree $t=\sum t_{i}$, so that there is an $a=\left(a_{1}, a_{2}, \cdots, a_{r}\right)$ in $A^{r}$ for which $q(a) \neq 0$. Let $E$ be the free nonassociative algebra without identity on $r$ generators, $z_{1}, z_{2}, \cdots, z_{r}$, and let $J$ be the ideal in $E$ generated by the set $J_{0}$ of all $f(e)$ in $E$, for some $f \in S$ and some $e \in E^{k}$, where $k$ is the number of indeterminates in $f$. Clearly $E / J$ is then an algebra satisfying $S$. On the other hand $E / J$ does not satisfy $q$ for the following reasons. The function $z_{i} \mapsto a_{i}$ has a unique extension to a homomorphism $\phi$ on $E$ onto the subalgebra $C$ of $A$ generated by the $a_{i}$. $C$, like $A$, satisfies $S$ but not $q$, and therefore $J C \operatorname{ker} \phi$. Then $C \cong(E / J) /(\operatorname{ker} \phi / J)$ is a homomorphic copy of $E / J$, so that $E / J$ cannot satisfy $q$. In fact, $q(z) \notin J$, where $z=\left(z_{1}, z_{2}, \cdots, z_{r}\right)$, since $q(a) \neq 0$ in $C$.

We will produce an enlargement $I$ of $J$ such that $E / I$ still fails to satisfy $q$ but can be shown to satisfy $p$. Of course $E / I$ satisfies $S$, since $J \subset I$, and so can be taken as the desired algebra $B$. It suffices to take $I=J+K$ where $K$ is the span (and an ideal) of all monomials in $E$ of degree greater than $t_{i}$ in $z_{i}$ for at least one $i=1,2, \cdots, r$.

To show that $E / I$ fails to satisfy $q$ we need the fact that $J$ has a basis consisting of homogeneous polynomials, for then we can conclude that $q(z) \notin I$. Suppose $q(z)=j(z)+k(z) \in I=J+K$. Then $k(z)$ is a linear combination of monomials of high degree in at least one variable, while each term in $q(z)$ is of low degree in each. Thus $q(z)$ $=\left(j_{1}(z)-k(z)\right)+k(z)$, where, since $J$ has a homogeneous basis, $j_{1}(z)$ and $k(z)$ both lie in $J$. But then $q(z)=j_{1}(z)$ lies in $J$, contrary to what we have observed. The existence of a homogeneous basis for $J$ follows from the homogeneity of the members of $S$ as follows. For each $f \in S$ and each $e \in E^{k}, f(e)$ is, by the bilinearity of multiplication in $E$, a sum $\sum_{j} f\left(e_{j}\right)$ in which each $e_{j}$ lies in $E^{k}$ and has coordinates that are monomials. Thus, the $f\left(e_{j}\right)$ are homogeneous and span the same subspace of $E$ as $J_{0}$ does. Now the ideal $J$ consists of all sums of products having a factor in the span of $J_{0}$, but each of these products is itself a sum of homogeneous members of $J$. Thus $J$ is spanned by polynomials homogeneous in each of the $z_{i}$.

To show that $E / I$ satisfies $p$, let $p$ be of the type $\left[n_{1}, n_{2}, \cdots, n_{m}\right]$ 
and degree $n=\sum n_{i}$. In case $t<n, p(e)$ is of degree no less than $n$, for each $e=\left(e_{1}, e_{2}, \cdots, e_{m}\right) \in E^{m}$, and so every term of $p(e)$ is of degree greater than $t_{i}$ in $z_{i}$ for some $i$. Thus $p(e) \in K \subset I$, and therefore $E / I$ satisfies $p$. In case $t=n$ we further distinguish the cases $m \leqq r$ and $m>r$. We will show that for each $e$ in $E^{m}, p(e)$ lies in $K$ whenever $m \leqq r$, while if $m>r$ then $p(e)$ is a sum of members of $K$ and polynomials obtained from $p$ by identifying variables in $p$ or in some partial linearization of $p$.

When $m=r$ we have $q \in\left[t_{1}, t_{2}, \cdots, t_{r}\right], p \in\left[n_{1}, n_{2}, \cdots, n_{r}\right]$, $q<p, \sum t_{i}=\sum n_{i}$, and $n_{k}<t_{k}$ for the least index $k$ at which $n_{k} \neq t_{k}$. But then there is an index $h>k$ for which $n_{h}>t_{h}$ and consequently $p(z)$ is of degree $n_{h}>t_{h}$ in $z_{h}$ and so lies in $K$. In fact for every $e$ in $E^{r}$, $p(e)$ is, as already observed, a sum of polynomials $p\left(e_{j}\right)$ in which each $e_{j}$ has monomial coordinates. Should some coordinate of some $e_{j}$ have total degree greater than one, $p\left(e_{j}\right)$ will consist of terms of high degree in some $z_{i}$ and so lie in $K$. However, if each coordinate of $e_{j}$ is just $\lambda_{i} z_{i}$ for some $i$ and some $\lambda_{i} \in F$, then $p\left(e_{j}\right)$ still consists of terms each of which is of high degree in some $z_{i}$. In case $m<r$ essentially the same argument holds: when each coordinate of $e_{j}=\left(e_{j 1}, e_{j 2}, \cdots, e_{j m}\right)$ is of the form $\lambda_{i} z_{i}, p\left(e_{j}\right)$ will have high degree in some $z_{i}$.

When $m>r$ we have again various circumstances under which $p(e)$ lies in $K$, along with the additional possibility, occurring when each coordinate of $e$ is a linear combination of the $z_{i}$ 's, that $p(e)$ is just a polynomial in $E$ obtained from $p$ by identifying variables in some partial linearization of $p$. Such a $p(e)$ is either of high degree in some $z_{i}$ and so lies in $K$ or else is of the same type as $q$, hence lower than that of $p$. In the latter case $S$ implies $p(e)$ by hypothesis. So every algebra that satisfies $S$ satisfies $p(e)$. Now $E / I$ satisfies $S$, hence satisfies $p(e)$, and therefore $E / I$ satisfies $p$ in all cases.

Because of Theorem 1 we are able to reduce, relative to a set $S$ and in a finite number of steps, every homogeneous polynomial to an irreducible one, and thus discover a short list of all polynomial identities that are, in some sense, worthy of study. This reducibility of polynomials is stated in the following corollary.

COROLlaRy. If a polynomial $p$ in $H$ is not irreducible with respect to a subfamily $S$ of $H$, then there is a polynomial $q<p$ obtained from $p$ by identifying variables in $p$ or in some partial linearization of $p$ that is irreducible.

Proof. Since $p$ is not irreducible it yields, by identification of variables in $p$ and in partial linearizations of $p$, a polynomial of lower type not implied by $S$. Should the latter also not be irreducible, there 
is a polynomial $q$ of least type so obtained from $p$ and not irreducible. But then there is a polynomial $q_{1}<q$ obtained from $q$ and hence from $p$ by identification of variables and not implied by $S$. Then $q_{1}$ is irreducible.

II. Low degree polynomials irreducible in anticommutative algebras. In what follows we shall assume that char $F \neq 2$. To make the list of irreducible polynomials brief we shall record just one of those of a common type that are equivalent in the presence of anticommutativity. For example, let $p_{\lambda_{\mu}}(x, y)=\lambda x y+\mu y x$, with $\lambda$ and $\mu$ in $F$. Now $p_{11}$ produces the anticommutative law, and $\left\{p_{11}, p_{\lambda_{\mu}}\right\} \Rightarrow p_{10}$ whenever $\lambda \neq \mu$. Obviously $p_{10} \Rightarrow p_{\lambda \mu}$ for all $\lambda$ and $\mu$, so, if we find $p_{10}$ is irreducible with respect to $p_{11}$ we will call it the only irreducible polynomial of type $[1,1]$.

The polynomials $x^{n}, x y$, and $(x y) x$ are irreducible with respect to $p_{11}$ because they produce, through linearization and identification of variables, no polynomial of lower type, only $x^{2}$, and only $x^{3}$, respectively, while $p_{11} \Rightarrow \phi, x^{2}$, and $x^{3}$. They are, however, uninteresting for various reasons: every anticommutative algebra satisfies $x^{n}$ when $n>1$, only trivial algebras satisfy $x$ or $x y$, and only nil alternative algebras satisfy $p_{11}$ and $(x y) x$. These last are nilpotent when they are finite dimensional.

THEOREM 2. With the preceding convention and exceptions the only homogeneous polynomials of degree no greater than four over a field of characteristic different from two that are irreducible with respect to $p_{11}(x, y)=x y+y x$ are

(i) $J(x, y, z)=(x y) z+(y z) x+(z x) y$,

(ii) $[(x y) x] x$,

(iii) $J(x, y, x y)$,

(iv) $\alpha(x y)(x z)+\beta\{[(x y) x] z-[(x z) x] y\}+\gamma\{[(x y) z] x-[(x z) y] x\}+$ $(\beta+\gamma)[(y z) x] x$, for some $\alpha, \beta$, and $\gamma$, not all zero, in $F$, and

(v) $J(x, y, z) w-J(w, x, y) z+J(z, w, x) y-J(y, z, w) x$.

Proof. The only irreducible cubics not already described are the multilinear ones, i.e., those of type $[1,1,1]$. In the presence of the anticommutative law these assume the form $J_{\lambda \mu}(x, y, z)=(x y) z$ $+\lambda(y z) x+\mu(z x) y$ for some $\lambda, \mu \in F$. By setting $x=y$, we find that $\left\{p_{11}, J_{\lambda \mu}\right\} \Rightarrow(\lambda-\mu)(x z) x$. However, $p_{11} \neq(x z) x$ as the example of the Lie algebra of Euclidean 3-space shows: $(i j) i=j \neq 0$. So the irreducibility of $J_{\lambda \mu}$ implies that $\lambda=\mu$. Again, setting $x=z$ in $J_{\lambda \mu}$ we see that $\left\{p_{11}, J_{\lambda_{\mu}}\right\} \Rightarrow(1-\lambda)(x y) x$ and consequently that $\lambda=1$. Conversely, $J=J_{11}$ is irreducible, since it produces, through identification of 
variables, only the polynomials $x^{3}$ and $x^{2} z+(x z) x+(z x) x$, both of which are implied by $p_{11}$.

In an anticommutative algebra every polynomial of type $[3,1]$ reduces to $[(x y) x] x$ which is irreducible since it yields only $x^{4}$ of lower type, the latter already implied by $p_{11}$.

The typical polynomial of type $[2,2]$ is $m_{\lambda}(x, y)=[(x y) x] y$ $+\lambda[(x y) y] x$ which produces, by identification of three of the variables in its linearized form, $x^{3} y+[(x y) x] x+\lambda\left\{\left(x^{2} y\right) x+[(x y) x] x\right\}$ so that $\left\{p_{11}, m_{\lambda}\right\} \Rightarrow(1+\lambda)[(x y) x] x$. Now $p_{11} \neq[(x y) x] x$, since $[(i j) i] i=-k \neq 0$ in $E^{3}$, so that $m_{\lambda}$ is irreducible only if $\lambda=-1$. Conversely, $m_{-1}$ is irreducible because it yields only $x^{4}$ and polynomials of type [3, 1] each of whose terms has a factor $x^{2}$, all of which then are satisfied by anticommutative algebras. Observe that $m_{-1}(x, y)=J(x, y, x y)$ is the polynomial that provides the defining identity for extended Lie algebras [3].

Of type $[2,1,1], p(x, y, z)=\alpha(x y)(x z)+\beta[(x y) x] z+\gamma[(x y) z] x$ $+\delta[(x z) x] y+\epsilon[(x z) y] x+\eta[(y z) x] x$ is typical and yields, by identification of variables

(i) $(\beta+\delta)[(x y) x] y+(\gamma+\epsilon)[(x y) y] x$,

(ii) $(\delta+\epsilon+\eta)[(x z) x] x$, and

(iii) $(\beta+\gamma-\eta)[(x y) x] x$, of lower type than $p$.

Again, $p_{11} \neq[(x y) x] x$, so that $p$ is irreducible only if $\delta+\epsilon+\eta=\beta+\gamma-\eta$ $=0$, in which case $\gamma+\epsilon=-(\beta+\delta)$, and (i) becomes $(\beta+\delta) m_{-1}$. Now $p_{11} \neq m_{-1}$ as the free anticommutative algebra on two generators shows. Thus $p$ is irreducible only if $\delta+\epsilon+\eta=\beta+\gamma-\eta=\beta+\delta=0$. Then $\delta=-\beta, \epsilon=-\gamma$, and $\eta=\beta+\gamma$, so that $p$ becomes polynomial (iv) of the theorem. Now the only nonzero polynomials of lower type yielded by $p$ have factors $x^{2}$ in every term, and so $p$ is irreducible with respect to anticommutativity.

Identifying variables in the general multilinear homogeneous quartic,

$$
\begin{aligned}
q(x, y, z, w)= & \alpha_{1}(x y)(z w)+\alpha_{2}(y z)(x w)+\alpha_{3}(z x)(y w) \\
& +\left[\alpha_{4}(x y) z+\alpha_{5}(y z) x+\alpha_{6}(z x) y\right] w \\
& +\left[\alpha_{7}(x y) w+\alpha_{8}(y w) x+\alpha_{9}(w x) y\right] z \\
& +\left[\alpha_{10}(x z) w+\alpha_{11}(z w) x+\alpha_{12}(w x) z\right] y \\
& +\left[\alpha_{18}(y z) w+\alpha_{14}(z w) y+\alpha_{15}(w y) z\right] x,
\end{aligned}
$$

yields, among others, eight polynomials of lower type. Of type $[3,1]$ are

$$
\left[\left(\alpha_{5}+\alpha_{10}+\alpha_{13}\right)-\left(\alpha_{6}+\alpha_{11}+\alpha_{14}\right)\right][(x z) x] x,
$$




$$
\begin{gathered}
{\left[\left(\alpha_{4}+\alpha_{7}+\alpha_{15}\right)-\left(\alpha_{5}+\alpha_{8}+\alpha_{13}\right)\right][(x y) x] x, \text { and }} \\
{\left[\left(\alpha_{6}+\alpha_{9}+\alpha_{12}\right)-\left(\alpha_{4}+\alpha_{7}+\alpha_{10}\right)\right][(y x) y] y .}
\end{gathered}
$$

Of type $[2,2]$ are

(iv) $\left(\alpha_{5}-\alpha_{6}+\alpha_{8}-\alpha_{9}\right)[(x z) x] z+\left(\alpha_{10}-\alpha_{12}+\alpha_{13}-\alpha_{15}\right)[(x z) z] x$ and

(v) $\left(\alpha_{7}-\alpha_{8}+\alpha_{10}-\alpha_{11}\right)[(x y) x] y+\left(\alpha_{4}-\alpha_{6}+\alpha_{15}-\alpha_{14}\right)[(x y) y] x$.

Of type $[2,1,1]$ are

$$
\begin{aligned}
& \left(\alpha_{2}-\alpha_{3}\right)(x z)(x w)+\left(\alpha_{5}-\alpha_{6}\right)[(x z) x] w+\left(\alpha_{8}-\alpha_{9}\right)[(x w) x] z \\
& +\left(\alpha_{10}+\alpha_{13}\right)[(x z) w] x-\left(\alpha_{12}+\alpha_{15}\right)[(x w) z] x+\left(\alpha_{11}+\alpha_{14}\right)[(z w) x] x \\
& \left(\alpha_{1}-\alpha_{2}\right)(x y)(x w)+\left(\alpha_{4}-\alpha_{5}\right)[(x y) x] w+\left(\alpha_{11}-\alpha_{12}\right)[(x w) x] y \\
& +\left(\alpha_{7}-\alpha_{13}\right)[(x y) w] x+\left(\alpha_{14}-\alpha_{9}\right)[(x w) y] x+\left(\alpha_{8}-\alpha_{15}\right)[(y w) x] x
\end{aligned}
$$

and

$$
\begin{aligned}
& -\left(\alpha_{1}+\alpha_{3}\right)(x y)(x z)+\left(\alpha_{7}-\alpha_{8}\right)[(x y) x] z+\left(\alpha_{10}-\alpha_{11}\right)[(x z) x] y \\
& +\left(\alpha_{4}+\alpha_{15}\right)[(x y) z] x+\left(-\alpha_{14}-\alpha_{6}\right)[(x z) y] x+\left(\alpha_{5}+\alpha_{13}\right)[(y z) x] x
\end{aligned}
$$

Since $p_{11} \neq[(x y) x] x$, the irreducibility of $q$ requires that the coefficients of polynomials (i) through (iii) vanish and therefore that

$$
\begin{aligned}
& \alpha_{12}=\alpha_{4}-\alpha_{6}+\alpha_{7}-\alpha_{9}+\alpha_{10}, \\
& \alpha_{14}=\alpha_{5}-\alpha_{6}+\alpha_{10}-\alpha_{11}+\alpha_{13}, \\
& \alpha_{15}=-\alpha_{4}+\alpha_{5}-\alpha_{7}+\alpha_{8}+\alpha_{13} .
\end{aligned}
$$

But then polynomials (iv) and (v) become

(iv) $\left(\alpha_{5}-\alpha_{6}+\alpha_{8}-\alpha_{9}\right) m_{-1}$ and

(v) $\left(\alpha_{7}-\alpha_{8}+\alpha_{10}-\alpha_{11}\right) m_{-1}$, respectively, whose coefficients must vanish if $q$ is to be irreducible (since $p_{11} \neq m_{-1}$ ). Thus

$$
\begin{aligned}
\alpha_{9} & =\alpha_{5}-\alpha_{6}+\alpha_{8}, \\
\alpha_{11} & =\alpha_{7}-\alpha_{8}+\alpha_{10} .
\end{aligned}
$$

Now the polynomials (vi) through (viii) become

$$
\begin{aligned}
& \left(\alpha_{2}-\alpha_{3}\right)(x z)(x w)+\left(\alpha_{5}-\alpha_{6}\right)\{[(x z) x] w-[(x w) x] z\} \\
& +\left(\alpha_{10}+\alpha_{13}\right)\{[(x z) w] x-[(x w) z] x\}+\left(\alpha_{5}-\alpha_{6}+\alpha_{10}+\alpha_{13}\right)[(z w) x] x \\
& \left(\alpha_{1}-\alpha_{2}\right)(x y)(x w)+\left(\alpha_{4}-\alpha_{5}\right)\{[(x y) x] w-[(x w) x] y\} \\
& +\left(\alpha_{7}-\alpha_{13}\right)\{[(x y) w] x-[(x w) y] x\}+\left(\alpha_{4}-\alpha_{5}+\alpha_{7}-\alpha_{13}\right)[(y w) x] x
\end{aligned}
$$




$$
\text { (viii) } \begin{aligned}
& -\left(\alpha_{1}+\alpha_{3}\right)(x y)(x z)+\left(\alpha_{7}-\alpha_{8}\right)\{[(x y) x] z-[(x z) x] y\} \\
& +\left(\alpha_{5}-\alpha_{7}+\alpha_{8}+\alpha_{13}\right)\{[(x y) z] x-[(x z) y] x\}+\left(\alpha_{5}+\alpha_{13}\right)[(y z) x] x,
\end{aligned}
$$

respectively. But these are just the irreducible polynomial (iv) of the theorem, which is not implied by $p_{11}$, as the free anticommutative algebra on three generators shows. So $q$ is irreducible only if the coefficients in (vi) through (viii) vanish. Combining this information with (A) and (B) produces

$$
\begin{array}{ll}
\alpha_{1}=\alpha_{2}=\alpha_{3}=0 \\
\alpha_{i}=\beta \neq 0, & \text { for } i=4,5,6,10,11,12 \\
\alpha_{i}=-\beta, & \text { for } i=7,8,9,13,14,15
\end{array}
$$

but then $q$ becomes polynomial (v) of the theorem. That $q$ is irreducible follows as in the preceding cases.

We can sharpen the statement of Theorem 2 somewhat. By the corollary of Theorem 1 every polynomial in $H$ not irreducible with respect to $p_{11}$ implies a polynomial of lower type that $i s$. At the same time observe that each of the irreducible polynomials $x$ and $x y$ implies every polynomial listed in Theorem 2 , while $(x y) x$ implies $[(x y) x] x$, and $J$ implies $J(x, y, x y)$. The irreducible polynomials $x^{n}, n>1$, are satisfied by every anticommutative algebra of characteristic $\neq 2$. These remarks are summarized in the following statement.

THEOREM 3. Every anticommutative algebra over a field of characteristic different from two that satisfies a homogeneous identity of degree no higher than four not already implied by the anticommutative law satisfies one of the following:

(1) $[(x y) x] x$,

(2) $J(x, y, x y)$,

(3) $\alpha(x y)(x z)+\beta\{[(x y) x] z-[(x z) x] y\}+\gamma\{[(x y) z] x-[(x z) y] x\}$ $+(\beta+\gamma)[(y z) x] x$,

(4) $J(x, y, z) w-J(w, x, y) z+J(z, w, x) y-J(y, z, w) x$.

The polynomials (1) through (4) are written in ascending order of type, and $J$ has a type lower than that of (1). By definition of irreducibility, there is, for each polynomial $p$ in this list, an anticommutative algebra satisfying $p$ but not satisfying any polynomial lower in the list. In fact, a finite dimensional example of an algebra satisfying (4) but not $J$ is easy to produce once we observe that (4) is skew symmetric in the presence of anticommutativity, for then (4) will be satisfied by every 3-dimensional anticommutative algebra, while at least one such is known not to be Lie. 
Let $p$ be the polynomial (4) and let $\pi \in S_{4}$. To show that

$$
p(\pi(x), \pi(y), \pi(z), \pi(w))=\operatorname{sgn} \pi p(x, y, z, w)
$$

for all $x, y, z, w$ in an anticommutative algebra, it suffices to do so for the cases that $\pi$ is the transposition $(x y)$ and the cycle $(x y z w)$, for these generate $S_{4}$. By substitution, use of the anticommutative law, and the fact that $J$ is skew symmetric in anticommutative algebras, we conclude that $p$ is skew symmetric. Thus $p$ vanishes whenever two of its variables are replaced by the same element from such an algebra. Since $p$ is multilinear it will be satisfied by any algebra for which it is satisfied by a basis of the algebra. Thus, it is satisfied by every algebra of dimension $\leqq 3$, for then every replacement of variables in $p$ by basis elements necessitates using some basis element twice. The algebra $A$ with basis $\{x, y, z\}$ and multiplication table $x y=x, x z=y$, $y z=0, a b=-b a$ for all $a, b=x, y$, or $z$, is anticommutative, satisfies $p$, and has a derived series $A, A^{\prime}, A^{\prime \prime}, A^{\prime \prime \prime}=0$ in which $\operatorname{dim} A^{\prime}=2$ and $\operatorname{dim} A^{\prime \prime}=1$. It is well known [1] that no such algebra is a Lie algebra.

\section{BiBLIOGRAPHY}

1. Nathan Jacobson, Lie algebras, Interscience Tracts in Pure and Appl. Math., no. 10, Interscience, New York, 1962. MR 26 \#1345.

2. J. Marshall Osborn, Identities of non-associative algebras, Canad. J. Math. 17 (1965), 78-92. MR 31 \#3470.

3. A. A. Sagle, On simple extended Lie algebras over fields of characteristic zero, Pacific J. Math. 15 (1965), 621-648. MR 32 \#7612.

Illinois Institute of Technology, Chicago, Illinois 60616

University of New Hampshire, Durham, New Hampshire 03824 